\title{
SEARCH FOR PENTAQUARK STATES ON PROTON TARGET AT CLAS
}

\author{
R. DE VITA, \\ FOR THE CLAS COLLABORATION \\ Istituto Nazionale di Fisica Nucleare \\ via Dodecaneso 33, 16146 Genova, Italy \\ E-mail: devita@ge.infn.it
}

\begin{abstract}
Baryon states beyond the usual $q q q$ configuration has been searched for many years but no evidence was found until recently. The findings about a possible pentaquark state have driven a rebirth of the experimental activity in this field. A broad experimental program for the search for pentaquark states is presently in progress at Jefferson Lab with the CLAS detector. In this proceedings the results and perspective for experiments using proton targets are discussed.
\end{abstract}

\section{Physics Motivation}

The existance of baryon states that cannot be described in terms of three valence quark is not prohibited by QCD. Search for such states started already several decades ago but no positive results were obtained until first evidence for a $\mathrm{S}=+1$ baryon, called $\Theta^{+}$, was reported by the LEPS/Spring8 Collaboration based on the analysis of photoproduction data on a carbon target. ${ }^{1}$ This baryon can be interpreted as pentaquark state with minimal $u u d d \bar{s}$ configuration.

Since the first announcement, evidence for the state has been claimed in more than 10 published papers from medium energy to very high energy experiments, using a variety of probes and target. In the last months negative results were also reported by several experiments, mostly at high energy. Presently, there is no definite answer on the existence or nonexistence of the $\Theta^{+}$. This is clearly of utmost importance: if confirmed, this discovery would open a new chapter in hadron spectroscopy and would provide fundamental information on hadronic structure.

In this contribution I report on the results obtained at Jefferson Lab with the CLAS detector using a photon beam and a proton target. In addition I will discuss the ongoing program of second generation experiments 
2
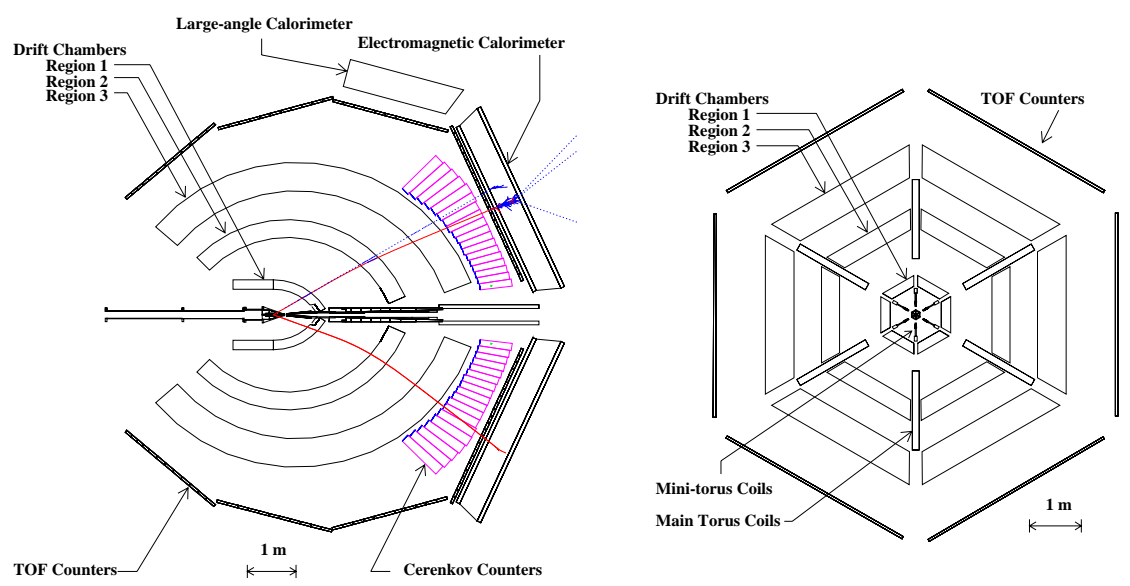

Figure 1. The CLAS detector. Left: Longitudinal cut along the beam line shows the different detector components. Right: Transverse cut through CLAS.

aimed at improving the statistical accuracy of the measurements by at least one order of magnitude.

\section{Experimental Setup}

The core of Jefferson Lab is the CEBAF accelerator (Continuous Electron Beam Accelerator Facility). This consists of two superconducting linacs connected by recirculation arcs. The superconductive cavities operate at a frequency of $1.497 \mathrm{GHz}$, resulting in a $2.0 \mathrm{~ns}$ duty cycle beam for the three experimental halls (Hall A, B, and C). The injected electrons can circulate up to five times reaching a maximum energy of $5.75 \mathrm{GeV}$.

In Hall $\mathrm{B}$, the electron beam is converted into a bremsstrahlung photon beam using a gold radiator located 20 meters upstream of the target. ${ }^{2}$ The photon energy is measured by detecting the scattered electron using a tagging system that operates over a range from $20 \%$ to $95 \%$ of the incident electron energy with a resolution of $10^{-3}$. The tagged photons, with rates between $10^{6}$ and $10^{7}$ per second, interact with a liquid hydrogen target located inside the CLAS detector (CEBAF Large Acceptance Spectrometer). The CLAS detector is shown in Figure 1. It is a magnetic spectrometer divided into six independent sectors. Each sector is equipped with three layers of drift chambers for tracking reconstruction, threshold Cherenkov counter for pion/electron discrimination, scintillators for timeof-flight measurements, and electromagnetic calorimeters for electrons and 
Table 1. Runs completed in Hall B at Jefferson Lab with the CLAS detector using photon beams and proton targets. The experimental conditions are summarized.

\begin{tabular}{|c|c|c|c|c|}
\hline Experiment & Year & Beam Energy & Target & $\int \mathcal{L}\left(\mathrm{pb}^{-1}\right)$ \\
\hline \hline g6a & 1998 & $4.1 \mathrm{GeV}$ & $\mathrm{LH}_{2}$ & $\sim 1.0$ \\
\hline g6b & 1999 & $5.5 \mathrm{GeV}$ & $\mathrm{LH}_{2}$ & $\sim 1.0$ \\
\hline g1c & 1999 & $1.9-3.1 \mathrm{GeV}$ & $\mathrm{LH}_{2}$ & $\sim 4.0$ \\
\hline g6c & 2000 & $5.7 \mathrm{GeV}$ & $\mathrm{LH}_{2}$ & $\sim 2.7$ \\
\hline
\end{tabular}

neutrals identification. The magnetic field produced by a six-coil toroidal magnet is oriented in such a way as to maintain the azimuthal angle of the scattered particles constant while changing only their polar angle.

The large acceptance of the detector allows the simultaneous measurement of several processes in a large kinematic domain. This capability is very important for the search of exotics, like the $\Theta^{+}$and its partners. In fact the production cross section for such particles are expected to be small, and the exotics signals may be easily hidden by known baryon production. Such background processes may be significantly reduced by selecting particular kinematics accessible with the CLAS detector.

In the last year, the existing CLAS data were reanalyzed to study possible evidence for pentaquark production. A summary of the different experiments with photon beam and proton target is given in Table 1.

\section{Results on Proton Target}

\subsection{Published Results}

The first evidence for the $\Theta^{+}$on proton target at CLAS was found in the analysis of the reaction $\gamma p \rightarrow \pi^{+} K^{+} K^{-} n .{ }^{4}$ The analysis was performed using the data recorded during the $g 6$ run. This data sets was accumulated in three different periods starting in 1998, with electron beam energy from 4.1 to $5.7 \mathrm{GeV}$ (see Table 1). The reaction was isolated detecting the three charged particles in the final state and selecting the unmeasured neutron using the missing mass technique. Production of known hyperons like the $\phi$ meson was suppressed applying cuts on the corresponding mass spectrum.

To understand the possible production mechanism for the $\Theta^{+}$and for the background, different possibilities were explored. For example the $\Theta^{+}$ may be produced through an intermediated $N^{*}$ resonance as shown in the top left diagram of Figure 2. Following this assumption events with a forward going $\pi^{+}$were selected, while events with a forward going $K^{+}$were rejected to reduce $t$-channel contribution. The final $n K^{+}$mass spectrum 

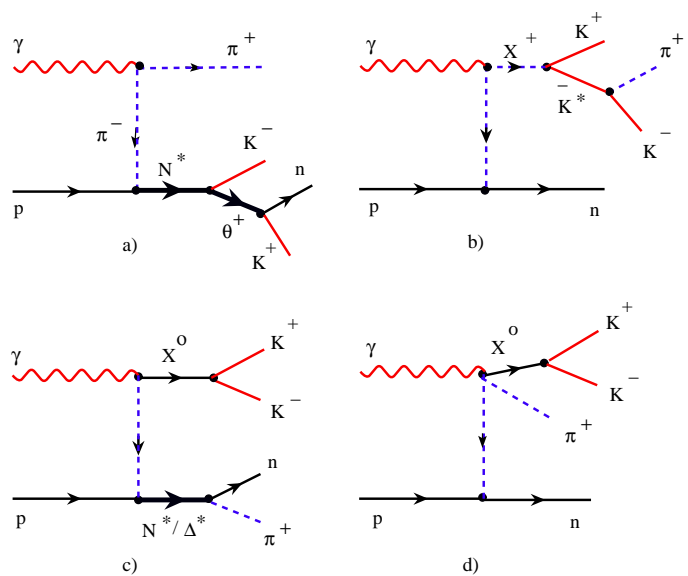

Figure 2. Diagrams that may contribute to the process $\gamma p \rightarrow \pi^{+} K^{-} n K^{+}$. The left top diagram contributes to $\Theta^{+}$production through intermediate $N^{*}$ excitation. The other diagrams represent background processes.

is shown in the left panel of Figure 3. A peak at a mass of $\sim 1.55 \mathrm{GeV}$ is observed over a smooth background. The spectrum was fitted with the sum of a gaussian function and of a background function obtained from simulations. A mean of $1550 \pm 10 \mathrm{MeV}, \Gamma=26 \pm 7 \mathrm{MeV}$, and a statistical significance of $7.8 \pm 1.0 \sigma$ for the observed structure were found.

To further study the background processes and to exclude systematic effects due to meson reflections in the $n K^{+}$mass spectrum, a full partial wave analysis of the $K^{+} K^{-} \pi^{+}$meson system was performed. This confirmed that the background is expected to be smooth and structureless.

If the $\Theta^{+}$production mechanism indeed involves the excitation of an intermediate $N^{*}$ resonance, this may appear in the $n K^{+} K^{-}$mass spectrum. This is shown in the right panel of Figure 3 for the events with effective $n K^{+}$mass between 1.54 and $1.58 \mathrm{GeV}$. An excess of events at a mass of $\sim 2.4 \mathrm{GeV}$ is observed while the partial wave analysis results shown by the line confirms a structureless background contribution. This result is consistent with the assumption of an intermediate $N^{*}$ contribution, but the statistics is at present too poor to reach any definite conclusion and further studies are needed.

\subsection{Ongoing Data Analysis}

In addition to the $g 6$ run period, other data sets have been analyzed looking for pentaquark states. The $g 1 c$ data set was used to study the reactions $\gamma p \rightarrow K^{+} \bar{K}^{0} n$ and $\gamma p \rightarrow p K^{+} K^{-}$, searching for evidence of $S=+1$ 

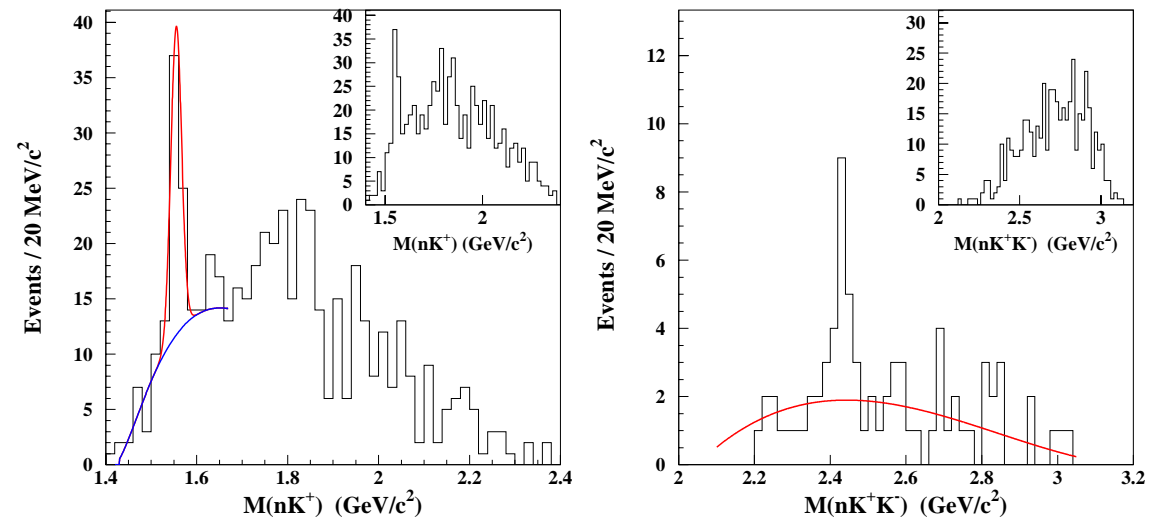

Figure 3. Left: Invariant mass distribution of $M\left(n K^{+}\right)$when all cuts are applied. The inset shows the $n K^{+}$mass distribution with only the $\cos \theta_{\pi^{+}}^{*}>0.8$ cut applied. Right: Mass distribution $M\left(K^{-} n K^{+}\right)$for events selected in the peak region of the graph on the left. The inset shows the distribution for events outside of the $\Theta^{+}$region.

resonances in the $K^{+} n$ and $K^{+} p$ invariant masses.

The first reaction was selected by detecting the final state charged particles, the $K^{+}$and the $\pi^{+} \pi^{-}$from the $\bar{K}^{0}$ decay, in CLAS. The neutron was not measured but was reconstructed using the missing mass technique, ensuring therefore the exclusivity of the final state. Figure 4 shows the quality of the channel identification: both $\bar{K}^{0}$ and $n$ are reconstructed within 1-2 $\mathrm{MeV}$ of the nominal mass value with small background. The events laying in the shaded region were selected for further analysis. This event sample is still dominated by the production of known hyperons decaying into the same final state. These include the production of $\Lambda$ excited states as for example $\gamma p \rightarrow K^{+} \Lambda^{*}(1520)$, or the production of $\Sigma \mathrm{s}$ in the reactions $\gamma p \rightarrow K^{+} \Sigma^{+} \pi^{-}$and $\gamma p \rightarrow K^{+} \Sigma^{-} \pi^{+}$. Figure 5 shows the $\Lambda^{*}(1520)$ and $\Sigma^{+}$peaks reconstructed as missing mass of $K^{+}$and the $K^{+} \pi^{-}$system. Events associated with these reactions were excluded by cutting on the corresponding masses. After such cuts, the $n K^{+}$invariant mass spectrum was constructed. After selecting events in which the $\bar{K}^{0}$ is emitted at backward angles, two structures with masses near $\sim 1525$ and $\sim 1575$ $\mathrm{MeV}$ were seen. However, the low statistics of the final event sample did not allow us to draw definitive conclusion on such structures.

The reaction $\gamma p \rightarrow p K^{+} K^{-}$was selected by detecting at least two of the three charged particles in CLAS and using the missing mass technique allows us to identify the third one. Two different topologies, $\gamma p \rightarrow p K^{+}\left(K^{-}\right)$ and $\gamma p \rightarrow(p) K^{+} K^{-}$, were analyzed while the topology with $p K^{-}$detected 
was dropped due to limited acceptance. Background contribution from known hyperons as the $\phi(1020)$ or $\Lambda^{*}(1520)$ were rejected with cuts on the corresponding masses. To further reduce the background contribution coming from other reactions and to maximize the signal to background ratio, angular and energy regions were selected where Monte Carlo simulations showed maximum sensitivity to the reactions of interest.
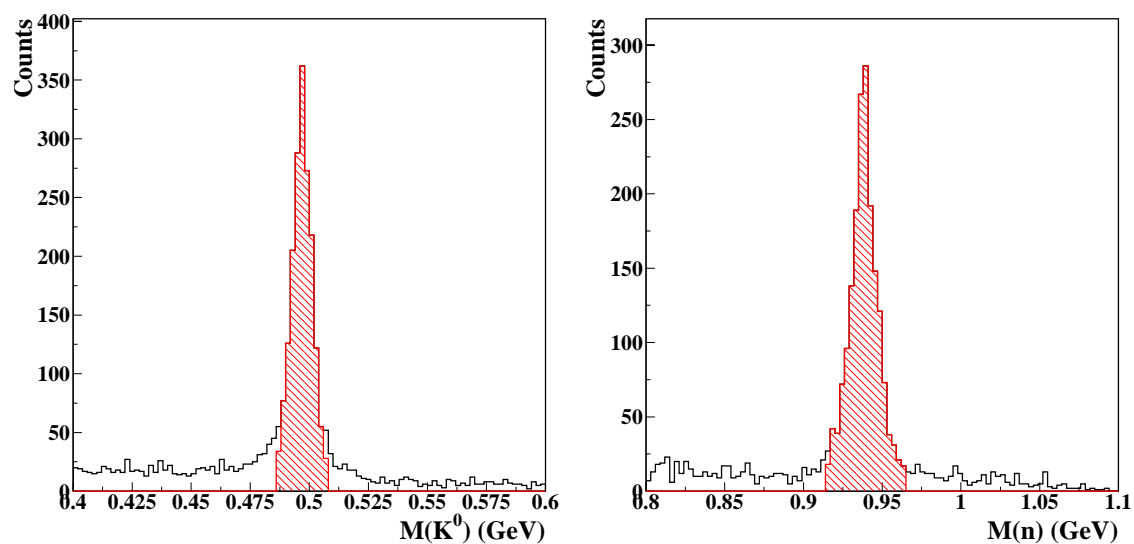

Figure 4. Final particle identification for the reaction $\gamma p \rightarrow K^{+} \bar{K}^{0} n$. The left plot shows the $\bar{K}^{0}$ mass spectrum reconstructed as invariant mass the $\pi^{+} \pi^{-}$system. The right plot shows the $K^{+} \pi^{+} \pi^{-}$missing mass.

After these additional cuts, the $p K^{+}$invariant mass spectrum showed a structure in the mass region around $1.58 \mathrm{MeV}$. However also in this case, the limited statistics did not allow us to reach definitive conclusions.

In either case, the much higher statistics of the new g11 experiment that is presently in the analysis stage, will allow more definite conclusions as to the existence and significance of these possibly new narrow structures.

Table 2. New experiments proposed in Hall B for the search of pentaquark states .

\begin{tabular}{|c|c|c|c|l|l|}
\hline Run & Beam & Energy & Target & Reaction & Status \\
\hline \hline $\mathrm{g} 11$ & $\gamma$ & $4.0 \mathrm{GeV}$ & $\mathrm{LH}_{2}$ & $\begin{array}{l}\gamma p \rightarrow \Theta^{+} \bar{K}^{0} \\
\gamma p \rightarrow \Theta^{+} K^{-} \pi^{+}\end{array}$ & Data Taking Completed \\
\hline $\mathrm{g} 12$ & $\gamma$ & $5.7 \mathrm{GeV}$ & $\mathrm{LH}_{2}$ & $\begin{array}{l}\gamma p \rightarrow \Theta^{+} K^{-} \pi^{+} \\
\gamma p \rightarrow \Theta^{+} \bar{K}^{0} \\
\gamma p \rightarrow K^{+} K^{-} \Xi^{-}\end{array}$ & To be scheduled \\
& & & & & \\
\hline
\end{tabular}



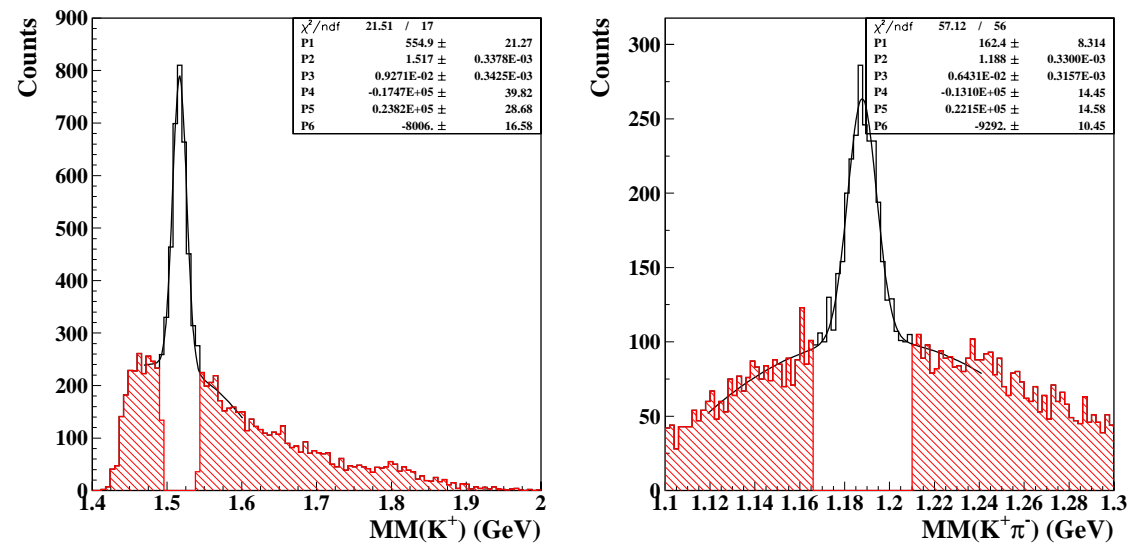

Figure 5. $K^{+}$and $K^{+} \pi^{-}$missing masses after the cuts on the $K^{+}$and $\bar{K}^{0}$, and $n$ masses. The $\Lambda^{*}(1520)$ and $\Sigma^{+}$peaks are clearly visible. The highlighted areas correspond to the events selected for further analysis.

\section{Perspectives and Future Plans}

The results obtained from the analysis of the existing data demonstrate the CLAS capability of detecting the reactions of interest for the pentaquark search with good resolution and limited background. The large acceptance of the detector allowed for fully identified final states while explicitly rejecting known background sources. However the available statistics do not exclude that the observed signals may be affected by statistical fluctuations, kinematic reflections, or some artifact of the data analysis.

A definitive answer about the existence of pentaquark states can be obtained only with new high statistics, high resolution experiments. For this purpose two new experiments on proton target have been recently approved for CLAS in Hall B at Jefferson Lab. The experimental conditions of this new runs are summarized in table 2.

The first experiment $\left(g_{11}\right)^{5}$ whose data taking was completed in July 2004 aims at establishing the phenomenology of the $\Theta^{+}$spectrum, e.g. determining in what production channels the $\Theta^{+}$is seen and what higher mass states are excited. ${ }^{6}$ The second experiment $\left(g_{12}\right)^{7}$ that will be scheduled in the near future will exploit the maximum available energy to study the $\Theta^{+}, \Xi$, and other pentaquark production measuring cross section, angular distribution, and decay distributions.

If the existence of the $\Theta^{+}$is confirmed, these new experiments will 

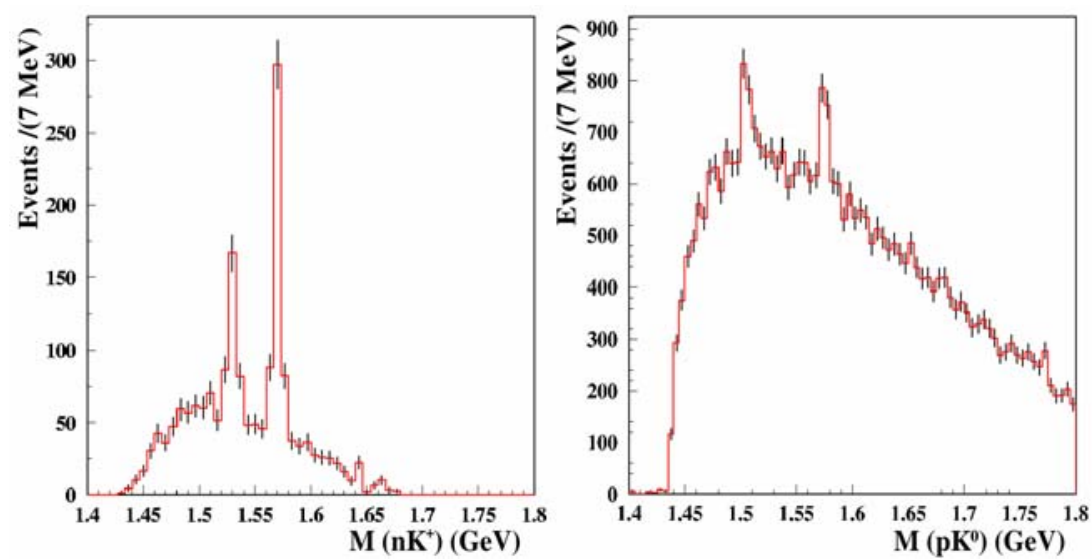

Figure 6. Expected statistical accuracy of the mass spectra for the reactions $\gamma p \rightarrow$ $\Theta^{+}\left(\Theta^{+*}\right) \bar{K}^{0}$, with $\Theta^{+}\left(\Theta^{+*}\right)$ decaying into $K^{+} n$ (left) and $p K^{0}$ (right) in the $g_{11}$ run. The background was estimated based on the existing data and the signal was simulated assuming a production cross section of $\sim 10 \mathrm{nb}$.

provide a solid foundation for a long term plan in pentaquark spectroscopy.

\section{Acknowledgments}

This work was supported by the Italian Istituto Nazionale di Fisica Nucleare, the French Centre National de la Research Scientifique, the French Commissariat à l'Energie Atomique, the U.S. Department of Energy, the U.S. National Science Foundation, and the Korean Science and Engineering Foundation. The Southeastern Universities Research Association (SURA) operates the Thomas Jefferson

National Accelerator Facility for the United States Department of Energy under contract DE-AC05-84ER40150.

\section{References}

1. T. Nakano et al., Phys. Rev. Lett. 91, 012002 (2003).

2. D. Sober et al., Nucl. Instr. and Meth. A440, 263 (2000).

3. B. Mecking et al., Nucl. Instr. and Meth. A503, 513 (2003).

4. V. Koubarovsky et al., Phys.Rev.Lett. 92, 032001 (2004).

5. M. Battaglieri, R. De Vita, V. Koubarovsky, et al. (CLAS), JLab experiment E-04-021.

6. M. Battaglieri, these proceedings.

7. J. Price, D. Weygand, et al. (CLAS), Jlab experiment E-04-017. 